\title{
Relations in Grassmann Algebra Corresponding to Three- and Four-Dimensional Pachner Moves
}

Igor G. KOREPANOV

Moscow State University of Instrument Engineering and Computer Sciences, 20 Stromynka Str., Moscow 107996, Russia

E-mail: paloff@ya.ru

Received May 15, 2011, in final form December 16, 2011; Published online December 18, 2011 http://dx.doi.org/10.3842/SIGMA.2011.117

\begin{abstract}
New algebraic relations are presented, involving anticommuting Grassmann variables and Berezin integral, and corresponding naturally to Pachner moves in three and four dimensions. These relations have been found experimentally - using symbolic computer calculations; their essential new feature is that, although they can be treated as deformations of relations corresponding to torsions of acyclic complexes, they can no longer be explained in such terms. In the simpler case of three dimensions, we define an invariant, based on our relations, of a piecewise-linear manifold with triangulated boundary, and present example calculations confirming its nontriviality.
\end{abstract}

Key words: Pachner moves; Grassmann algebras; algebraic topology

2010 Mathematics Subject Classification: 15A75; 55-04; 57M27, 57Q10; 57R56

\section{Introduction}

The main aim of this paper is to present some results of symbolic calculations, namely, new algebraic relations with anticommuting Grassmann variables and Berezin integral, corresponding naturally to Pachner moves in three and four dimensions. These results do not rely on any finished theory; they were found starting from considerations related to some unusual chain complexes introduced in our previous works, an then using just free search on a computer, some heuristics like parameter counting, some hardly explainable tricks, and a hope that interesting relations may exist. Our software included GAP [7], our own package PL [8], and Maxima [15].

The essential new feature of our algebraic relations is that, although they can be treated as deformations of already known relations corresponding to torsions of acyclic complexes, they can no longer be formulated and explained in such terms ${ }^{1}$.

As we have said, our calculations - computer experiments - belong to three- and, what seems the most interesting, four-dimensional topology. As the four-dimensional case is more complicated, we restrict ourself to presenting relations corresponding to Pachner moves $3 \rightarrow 3$ and $2 \rightarrow 4$ (the first of them proved in full on a computer, while the second is a conjecture confirmed by numerical ${ }^{2}$ calculations), and a (conjectured) formula for a "partial" manifold invariant - a Grassmann algebra element preserved by these moves, leaving for future work both the construction of a "full" invariant and its calculations for specific manifolds. In contrast with this, we define an actual invariant of a three-dimensional piecewise-linear manifold with triangulated boundary, based on our relations, and present example calculations confirming its nontriviality.

\footnotetext{
${ }^{1}$ Note also that in our previous works, the (simpler) relations corresponding to Pachner moves were derived using direct calculations as well, using only some "partial" theoretical considerations.

${ }^{2}$ As opposed to symbolic.
} 

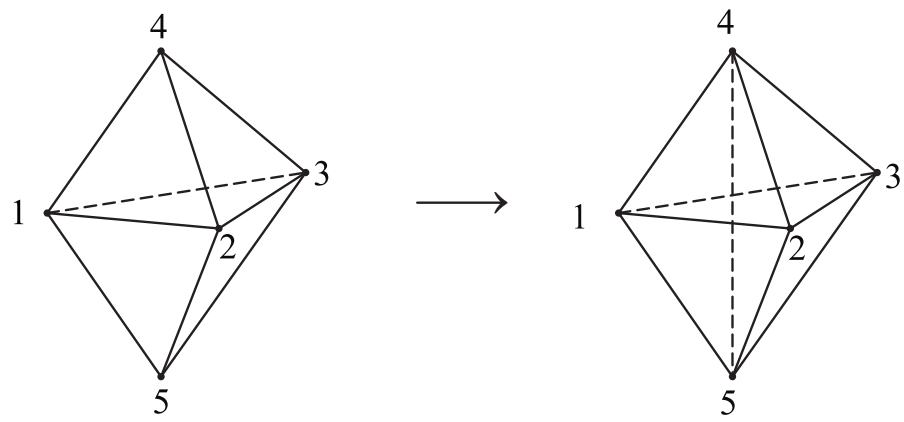

Figure 1. Pachner move $2 \rightarrow 3$.

This introduction continues with a brief reminder of what Pachner moves and Grassmann algebras are, in Subsections 1.1 and 1.2 respectively. Then, we explain the organization of the (main part of the) paper in Subsection 1.3.

\subsection{Pachner moves and algebraic relations}

Pachner moves [16] (see also a very good educational text [12]) are elementary rebuildings of a manifold triangulation. Their importance is due to the fact that any triangulation of a closed piecewise-linear (PL) manifold can be transformed into any other triangulation by a sequence of these moves, of which, moreover, there exists only a finite number in any manifold dimension. Thus, if we have algebraic formulas whose structure corresponds, in some natural sense, to all Pachner moves in a given dimension, then there is a big hope that we will be able - maybe with the help of some additional tools - to build a manifold invariant based on such formulas. Also, some experience shows that if there is a formula corresponding to just one Pachner move, then it makes a strong sense to search for more formulas for other moves.

Some modifications are to be done if we consider manifolds with boundary. Pachner proves that, in this case, any triangulation can be transformed into any other triangulation by a sequence of shellings and inverse shellings. Each of these operations affects the boundary and changes its triangulation. If we want, however, to construct an invariant of a manifold with a fixed boundary triangulation - and this is what we do in Section 4 of the present paper - we must choose another way. Namely, we consider relative Pachner moves, that is, moves not changing the boundary triangulation. The resulting invariant depends thus on the way the manifold is glued to its boundary ${ }^{3}$.

\subsubsection{Pachner moves in three dimensions}

There are four Pachner moves in three dimensions: $2 \leftrightarrow 3$ and $1 \leftrightarrow 4$.

Pachner move $2 \rightarrow 3$ is an elementary rebuilding of a 3 -manifold triangulation, which replaces two adjacent tetrahedra (1234) and (1235) (where of course (1234) is the tetrahedron with vertices 1, 2, 3 and 4, and so on) with three tetrahedra (1245), (1345) and (2345) occupying the same place in the manifold, as in Fig. 1.

Pachner move $1 \rightarrow 4$ adds a new vertex 5 inside a tetrahedron (1234) and replaces it with tetrahedra (1235), (1245), (1345) and (2345), see Fig. 2.

Two other moves are their inverses.

Remark 1. Strictly speaking, our triangulations are not exactly like in [12]: we allow using different simplices having the same boundary components, see Fig. 4 below for a good example.

\footnotetext{
${ }^{3}$ Recall that, for instance, in three dimensions there are many ways to glue a filled pretzel to its boundary.
} 

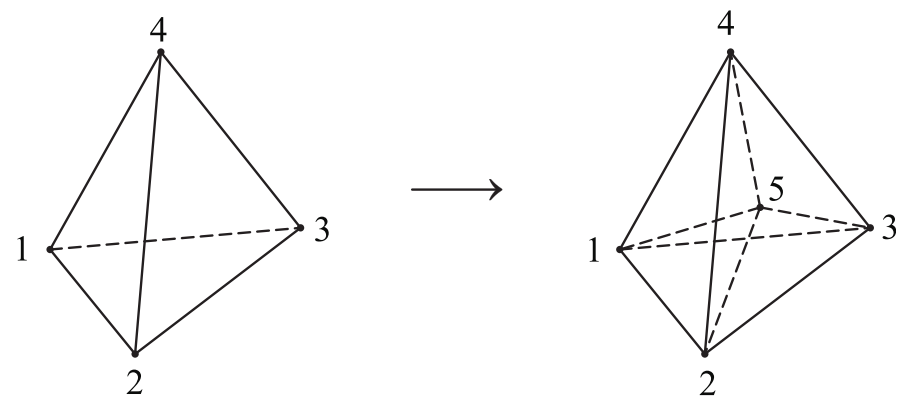

Figure 2. Pachner move $1 \rightarrow 4$.

Such triangulations are sometimes called "noncombinatorial" - a not very appropriate term, because combinatorics is exactly what we widely use, in particular, in our computer package PL [8]. Nevertheless, all our operations can be quite easily translated into the language of [12], see, for instance, again [5, Section 2].

\subsubsection{Pachner moves in four dimensions}

There are five Pachner moves in four dimensions: $3 \rightarrow 3,2 \leftrightarrow 4$ and $1 \leftrightarrow 5$.

In Section 3, we will be dealing with Pachner moves $3 \rightarrow 3$ and $2 \rightarrow 4$, their descriptions are given there in Subsections 3.2.1 and 3.2.2 respectively.

In this paper, we do not present formulas for a move $1 \rightarrow 5$, leaving this for further work. We would like only to explain here that this move consists in adding a new vertex 6 inside a given four-simplex (12345) and joining this new vertex with the boundary of (12345). This leads to the latter being divided into five tetrahedra (12346), (12356), (12456), (13456), and (23456).

\subsection{Grassmann algebras and Berezin integral}

A Grassmann algebra over a field $\mathbb{F}$ - for which we can take in this paper any field of characteristic $\neq 2$ - is an associative algebra with unity, having generators $a_{i}$ and relations

$$
a_{i} a_{j}=-a_{j} a_{i}
$$

As this implies for $i=j$ that $a_{i}^{2}=0$, any element of a Grassmann algebra is a polynomial of degree $\leq 1$ in each $a_{i}$. For a given Grassmann monomial, by its degree we understand its total degree in all Grassmann variables; if an element of Grassmann algebra includes only monomials of odd degrees, it is called odd; if it includes only monomials of even degrees, it is called even.

The exponent is defined by the standard Taylor series. For example,

$$
\exp \left(a_{1} a_{2}\right)=1+a_{1} a_{2} \text {. }
$$

If at least one of $\varphi_{1}$ and $\varphi_{2}$ is even, then

$$
\exp \left(\varphi_{1}\right) \exp \left(\varphi_{2}\right)=\exp \left(\varphi_{1}+\varphi_{2}\right) .
$$

The Berezin integral [2] is an $\mathbb{F}$-linear operator in a Grassmann algebra defined by equalities

$$
\int \mathrm{d} a_{i}=0, \quad \int a_{i} \mathrm{~d} a_{i}=1, \quad \int g h \mathrm{~d} a_{i}=g \int h \mathrm{~d} a_{i},
$$

if $g$ does not depend on $a_{i}$ (that is, generator $a_{i}$ does not enter the expression for $g$ ); multiple integral is understood as iterated one, according to the following model:

$$
\iint a b \mathrm{~d} b \mathrm{~d} a=\int a\left(\int b \mathrm{~d} b\right) \mathrm{d} a=1 .
$$


The left derivative $\partial / \partial a$ w.r.t. a Grassmann generator $a$ for a monomial $f$ is defined as follows: if $f$ does not contain $a$, then $\partial f / \partial a=0$, otherwise bring $a$ to the left using commutation relations (1) and strike it out.

Remark 2. A curious feature of Grassmann-Berezin calculus is that the integral is the same operation as the right derivative (defined in obvious analogy with the left one). Nevertheless, using the two names for one operation makes sense because sometimes this is an analogue of the usual "commutative" integral, and sometimes - of the derivative. Moreover, it may turn out that this feature makes Grassmann-Berezin calculus a powerful tool in constructing unusual algabraic structures.

\subsection{Organization of the paper}

A "theorem" in this paper is a statement proved either in a traditional way, or using a computer and software for symbolic calculations. A "conjecture" is a statement whose correctness raises practically no doubt but which has not been strictly proved. For instance, this can be a formula whose validity has been checked for some arbitrarily chosen set(s) of values of indeterminates, while checking it without assigning numerical values to indeterminates was beyond the available computational powers. Theorems and conjectures are numbered consecutively; "partial verification" of a conjecture corresponds to the "proof" of a theorem.

Below:

- in Section 2, we present our Grassmann algebraic relations corresponding to Pachner moves in three dimensions,

- in Section 3, we present similar relations corresponding to Pachner moves $3 \rightarrow 3$ and $2 \leftrightarrow 4$ in four dimensions,

- in Section 4, we return to the three-dimensional case and consider an example of invariant of three-manifolds with triangulated boundary, showing its nontriviality,

- in Section 5, we briefly discuss our results and further research.

\section{Three dimensions: relations corresponding to moves $2 \leftrightarrow 3$ and $1 \leftrightarrow 4$}

\subsection{Recalling the "undeformed" relations}

\subsubsection{The chain complex}

The starting point for the invariants - field theory amplitudes - introduced in this paper, is a particular - "scalar" - case of the theory exposed in paper [3]. Namely, we begin with the following chain complex built for a triangulated orientable three-manifold $M$ with boundary:

$$
0 \longrightarrow \mathbb{C}^{N_{0}^{\prime}} \stackrel{f_{2}}{\longrightarrow} \mathbb{C}^{N_{2}} \stackrel{f_{3}}{\longrightarrow} \mathbb{C}^{2 N_{3}} \stackrel{f_{4}}{\longrightarrow} \mathbb{C}^{N_{0}^{\prime}} \longrightarrow 0 .
$$

Here $N_{0}^{\prime}$ is the number of inner vertices in $M$, while $N_{2}$ - the number of all 2-faces, and $N_{3}-$ the number of all tetrahedra.

We assume that all vertices in $M$ are numbered from 1 to their total number $N_{0}$, and we ascribe "coordinates" $\zeta_{1}, \ldots, \zeta_{N_{0}}$ to them. These are arbitrary complex numbers with the only condition

$$
\zeta_{i} \neq \zeta_{j} \quad \text { for } \quad i \neq j \text {. }
$$


We will also use notation

$$
\zeta_{i j} \stackrel{\text { def }}{=} \zeta_{i}-\zeta_{j}
$$

Remark 3. The numbers $k$ of mappings $f_{k}$ in (5) begin from 2 and not 1 in order to make them consistent with similar complexes that include two more mappings: $f_{1}$ on the left and $f_{5}$ on the right, see, e.g., [4, formula (5)]. In this paper, however, we do not use complexes longer than (5).

Both spaces $\mathbb{C}^{N_{0}^{\prime}}$ in (5) consist, by definition, of column vectors whose components, denoted $u_{i}$ for the left-hand space and $v_{i}$ for the right-hand space, are in one-to-one correspondence with inner vertices $i$. More formally, each of these spaces is a space over $\mathbb{C}$ with inner vertices as its basis.

Spaces $\mathbb{C}^{N_{2}}$ and $\mathbb{C}^{2 N_{3}}$ are a bit more complicated. To explain them, we begin with two auxiliary spaces: $W_{2}$ whose basis is formed of all pairs $(s, i)$, where $s$ is a 2 -face and $i \in s$ - its vertex, and $W_{3}$ whose basis is formed of all pairs $(r, i)$, where $r$ is a tetrahedron and $i \in r-$ its vertex. Thus, $\operatorname{dim} W_{2}=3 N_{2}$ and $\operatorname{dim} W_{3}=4 N_{3}$. We use notations like $x_{s, i}$ or $y_{r, i}$ for coordinates of a vector $x \in W_{2}$ or $y \in W_{3}$.

Then we introduce space $V_{2} \subset W_{2}$ consisting of vectors whose coordinates obey

$$
\begin{aligned}
& x_{s, i}+x_{s, j}+x_{s, k}=0, \\
& \zeta_{i} x_{s, i}+\zeta_{j} x_{s, j}+\zeta_{k} x_{s, k}=0
\end{aligned}
$$

for every 2-face $s$ with vertices $i, j$ and $k$, and similarly space $V_{3} \subset W_{3}$ consisting of vectors whose coordinates obey

$$
\begin{aligned}
& y_{r, i}+y_{r, j}+y_{r, k}+y_{r, \ell}=0, \\
& \zeta_{i} y_{r, i}+\zeta_{j} y_{r, j}+\zeta_{k} y_{r, k}+\zeta_{\ell} y_{r, \ell}=0
\end{aligned}
$$

for every tetrahedron $r$ with vertices $i, j, k$ and $\ell$.

Thus, a vector $x \in V_{2}$ is determined by specifying just one its coordinate in each 2-face $s$, and assuming that $i<j<k$, we will take coordinate $x_{s, i}$ for that. The space $\mathbb{C}^{N_{2}}$ consists, by definition, of column vectors whose coordinates are these $x_{s, i}$ for all $s$.

Similarly, a vector $y \in V_{3}$ is determined by specifying just two of its coordinates in each tetrahedron $r$, and assuming that $i<j<k<\ell$, we will take $y_{r, i}$ and $y_{r, j}$ for that. The space $\mathbb{C}^{2 N_{3}}$ consists, by definition, of column vectors whose coordinates are these $y_{r, i}$ and $y_{r, j}$ for all $r$.

Linear mapping $f_{2}$ makes, by definition, the following $x_{s, i}$ from given $u_{i}$ :

$$
f_{2}: x_{s, i}=\left(\zeta_{i j}^{-1}-\zeta_{i k}^{-1}\right) u_{i}-\zeta_{i j}^{-1} u_{j}+\zeta_{i k}^{-1} u_{k},
$$

where 2 -face $s$ has vertices $i<j<k$.

Linear mapping $f_{3}$ makes, by definition, the following $y_{r, i}$ from given $x_{s, i}$ :

$$
f_{3}:\left\{\begin{array}{l}
y_{r, i}=x_{(i j k), i}-x_{(i j \ell), i}+x_{(i k \ell), i}, \\
y_{r, j}=x_{(i j k), j}-x_{(i j \ell), j}-x_{(j k \ell), j}
\end{array}\right.
$$

where tetrahedron $r$ has vertices $i<j<k<\ell$, and by $(i j k)$ and so on we denote the 2 -faces of $r$ containing the indicated vertices.

Convention 1. In general, when we write an $n$-simplex in this paper as $\left(i_{0} \ldots i_{n}\right)$, the (numbers of) its vertices are ordered so that $i_{0}<\cdots<i_{n}$, if other ordering is not indicated explicitly. 
To define linear mapping $f_{4}$, we must fix an orientation of $M$, i.e., a consistent orientation of all its tetrahedra. This results in ascribing a sign

$$
\epsilon_{r}= \pm 1
$$

to each tetrahedron $r$ with vertices $i<j<k<\ell$ in the following way: $\epsilon_{r}=1$ if the orientation of $r$ determined by the order $i, j, k, \ell$ of vertices coincides with the mentioned consistent orientation, and $\epsilon_{r}=-1$ otherwise. Mapping $f_{4}$ makes, by definition, the following $v_{i}$ from given $y_{r, i}$ :

$$
f_{4}: v_{i}=\sum_{r \ni i} \epsilon_{r} y_{r, i}
$$

the sum goes, of course, over all tetrahedra containing vertex $i$.

Theorem 1. The chain (5) of vector spaces and linear mappings defined as above is indeed a chain complex, i.e.,

$$
f_{4} \circ f_{3}=0, \quad f_{3} \circ f_{2}=0 .
$$

Proof. Theorem 1 can be proved by direct calculations. For a conceptual explanation of the origin of (5), see [3, Subsection 3.2].

\subsubsection{Invariants from Reidemeister torsions}

We want to calculate some Reidemeister torsions for chain complex (5). The complex (5) as it is will, however, never be acyclic for a manifold with non-empty boundary. This is because its algebraic Euler characteristic is $-N_{2}+2 N_{3} \neq 0$. To be more exact, in the case of non-empty boundary $N_{2}>2 N_{3}>N_{2}^{\prime}$, where $N_{2}^{\prime}$ is the number of inner 2-faces.

Actually, this allows us to introduce not one but many torsions. First, we take an ordered subset $\mathcal{C}$ of boundary faces of cardinality $\# \mathcal{C}=2 N_{3}-N_{2}^{\prime}$. Second, we consider, instead of $\mathbb{C}^{N_{2}}$, its subspace $\left(V_{2}\right)_{\mathcal{C}}$ consisting of those vectors whose coordinates corresponding to boundary faces outside $\mathcal{C}$ are zero. We assume also that the coordinates corresponding to inner edges go first and are ordered in the same fixed way for all $\mathcal{C}$, and then go the coordinates belonging to $\mathcal{C}$ and ordered also as $\mathcal{C}$. Third, we define a new complex - a subcomplex of (5) - by replacing $\mathbb{C}^{N_{2}}$ with $\left(V_{2}\right)_{\mathcal{C}}$ and restricting naturally linear mappings $f_{2}$ and $f_{3}$ - just taking their submatrices corresponding to $\left(V_{2}\right)_{\mathcal{C}}$.

It can be checked that Theorem 1 remains valid for this new chain complex corresponding to the set $\mathcal{C}$, and we define its Reidemeister torsion ${ }^{4}$ in a standard way as

$$
\tau_{\mathcal{C}}=\frac{\left(\operatorname{minor} f_{3}\right)_{\mathcal{C}}}{\operatorname{minor} f_{2} \operatorname{minor} f_{4}}
$$

where the minors are chosen according to the rules for a matrix $\tau$-chain, see [17, Subsection 2.1]. Moreover, we can take the the same minors of both $f_{2}$ and $f_{4}$ for all $\mathcal{C}$, and this is reflected in (14) by writing the subscript $\mathcal{C}$ only at (minor $\left.f_{3}\right)$.

Remark 4. Of course, for some $\mathcal{C}$ 's, both $\left(\text { minor } f_{3}\right)_{\mathcal{C}}$ and $\tau_{\mathcal{C}}$ will vanish, or, speaking more strictly, there will be no $\tau$-chain.

\footnotetext{
${ }^{4}$ This construction can be also interpreted in terms of torsions for chain complexes with nonvanishing homologies, see [17, Subsection 3.1]. We leave this as an exercise for the reader, just mentioning that a subset $\mathcal{C}$ determines a basis in the homology space corresponding to the middle term of the complex conjugate to (5), i.e., with arrows reversed and matrices $f_{2}, f_{3}, f_{4}$ transposed.
} 
Now we introduce the following quantities, where the letter $I$ stays for "invariant", and the superscript (0) is to emphasize that these are our "old" invariants, to be deformed soon:

$$
I_{\mathcal{C}}^{(0)}=\frac{\prod_{\substack{\text { inner } \\ 2 \text {-faces } s}} \zeta_{s_{2} s_{3}}}{\prod_{\substack{\text { inner } \\ \text { edges } \ell}} \zeta_{\ell_{1} \ell_{2}} \prod_{\substack{\text { all } \\ \text { tetrahedra } r}} \zeta_{r_{3} r_{4}}} \cdot \tau_{\mathcal{C}},
$$

where we use the following notations:

- $\ell_{1}$ and $\ell_{2}$ are the vertices of an inner edge $\ell$ taken in the increasing order: $\ell_{1}<\ell_{2}$,

- similarly, $s_{1}<s_{2}<s_{3}$ are the vertices of an inner 2-face $s$, and

- $r_{1}<r_{2}<r_{3}<r_{4}$ - the vertices of a tetrahedron $r$.

Theorem 2. The values (15) for all $\mathcal{C}$ form a multicomponent invariant of manifold $M$ with a fixed boundary triangulation, defined up to an overall (the same for all $\mathcal{C}$ ) sign.

Proof. To prove that $I_{\mathcal{C}}^{(0)}$, for a given $\mathcal{C}$, is a manifold invariant, it is enough to prove its invariance under:

(i) a change of order of inner vertices,

(ii) a Pachner move $2 \leftrightarrow 3$,

(iii) a Pachner move $1 \leftrightarrow 4$.

For items (ii) and (iii), we refer the reader to [3, Theorem 4], where this is proved in a more general situation ${ }^{5}$.

To prove $(\mathrm{i})^{6}$, we note that a change of vertex order implies the corresponding change of bases in spaces $V_{2}$ and $V_{3}$. To see the change of Reidemeister torsion, we must calculate determinants of transition matrices between bases in $V_{2}$ and $V_{3}$, or their inverses - ratios between exterior products of all new and all old coordinates in the corresponding space:

$$
\frac{\bigwedge\left(x_{s, i}\right)_{\text {new }}}{\bigwedge\left(x_{s, i}\right)_{\text {old }}} \quad \text { and } \quad \frac{\bigwedge\left(y_{r, i}\right)_{\text {new }}}{\bigwedge\left(y_{r, i}\right)_{\text {old }}}
$$

As one can deduce from (8) and (9) such relations as, for instance,

$$
\frac{x_{s, j}}{x_{s, i}}=-\frac{\zeta_{i k}}{\zeta_{j k}} \quad \text { and } \quad \frac{y_{r, k} \wedge y_{r, \ell}}{y_{r, i} \wedge y_{r, j}}=\frac{\zeta_{i j}}{\zeta_{k \ell}}
$$

it is not hard to check that the invariance of (15) really holds.

As for the sign of each $I_{\mathcal{C}}^{(0)}$, it is not determined uniquely because of arbitrariness of ordering basis vectors in our vector spaces. It can be easily seen, however, that any change in such ordering makes the same effect on the sign of every $I_{\mathcal{C}}^{(0)}$ : the only basis vectors that differ in two complexes corresponding to two $\mathcal{C}$ 's belong to these $\mathcal{C}$ 's, and their order is fixed because the $\mathcal{C}$ 's are ordered. Also, any possible sign ambiguities in the above transformations (i), (ii) and (iii) affect the signs of all $I_{\mathcal{C}}^{(0)}$ in the same way. This proves that all $I_{\mathcal{C}}^{(0)}$ are determined up to one overall sign.

\footnotetext{
${ }^{5}$ In the formulation of [3, Theorem 4], the boundary $\partial M$ is assumed to be one-component. This, however, is not used in the proof. The point is that, actually, (19) vanishes for multicomponent $\partial M$. In this paper we, nevertheless, do not put away the multicomponent case, because our aim is to introduce a deformation of (19) which may behave differently.

${ }^{6}$ It must be admitted that the (more general) analogue of (i) should have been proven also already in [3].
} 


\subsubsection{Invariants made from Reidemeister torsions in terms of Grassmann algebra}

We put in correspondence to each unoriented 2-face $s$ in the triangulation a Grassmann generator $a_{s}$, and to each unoriented ${ }^{7}$ tetrahedron $r$ two Grassmann generators $b_{r}^{(1)}$ and $b_{r}^{(2)}$.

We denote $\mathbf{a}$ the column vector made of all $a_{j}$, and $\mathbf{b}$ the column vector made of all $b_{r}^{(1)}$ and $b_{r}^{(2)}$.

Definition 1. For a tetrahedron $r$, we introduce its Grassmann weight as follows:

$$
W_{r}=\exp \Phi_{r},
$$

where

$$
\Phi_{r}=\left(\begin{array}{ll}
b_{r}^{(1)} & b_{r}^{(2)}
\end{array}\right)\left(\begin{array}{cccc}
1 & -1 & 1 & 0 \\
-\zeta_{r_{2} r_{3}}^{-1} \zeta_{r_{1} r_{3}} & \zeta_{r_{2} r_{4}}^{-1} \zeta_{r_{1} r_{4}} & 0 & -1
\end{array}\right)\left(\begin{array}{c}
a_{\left(r_{1} r_{2} r_{3}\right)} \\
a_{\left(r_{1} r_{2} r_{4}\right)} \\
a_{\left(r_{1} r_{3} r_{4}\right)} \\
a_{\left(r_{2} r_{3} r_{4}\right)}
\end{array}\right) .
$$

In (17), $r_{1}, r_{2}, r_{3}, r_{4}$ are the vertices of $r$ in the increasing order; $\left(r_{1} r_{2} r_{3}\right)$ and the like are the faces of $r$ having corresponding vertices. The $2 \times 4$ matrix in the r.h.s. is a block of which matrix $f_{3}$ is built, in accordance with (11), (8) and (9).

Definition 2. For every inner vertex $i$, we introduce the following vertex-face differential operator in our Grassmann algebra:

$$
d_{i}^{\mathbf{a}}=\sum_{2 \text {-faces } s \ni i} d_{s, i}^{\mathbf{a}}
$$

where

$$
d_{s, i}^{\mathbf{a}}= \begin{cases}\left(1 / \zeta_{s_{1} s_{2}}-1 / \zeta_{s_{1} s_{3}}\right) \partial / \partial a_{s} & \text { if } i=s_{1}, \\ \left(-1 / \zeta_{s_{1} s_{2}}\right) \partial / \partial a_{s} & \text { if } i=s_{2}, \\ \left(1 / \zeta_{s_{1} s_{3}}\right) \partial / \partial a_{s} & \text { if } i=s_{3}\end{cases}
$$

$s_{1}, s_{2}, s_{3}$ are the vertices of $s$ in the increasing order. The coefficients at $\partial / \partial a_{s}$ are matrix elements of matrix $f_{2}$, in accordance with (10).

Definition 3. Also, we introduce one more operator for every inner vertex $i$, the vertextetrahedron differential operator:

$$
d_{i}^{\mathbf{b}}=\sum_{\text {tetrahedra } r \ni i} d_{r, i}^{\mathbf{b}}
$$

where

$$
d_{r, i}^{\mathbf{b}}= \begin{cases}\epsilon_{r} \partial / \partial b_{r}^{(1)} & \text { if } i=r_{1}, \\ \epsilon_{r} \partial / \partial b_{r}^{(2)} & \text { if } i=r_{2}, \\ \epsilon_{r}\left(-\left(\zeta_{r_{1} r_{4}} / \zeta_{r_{3} r_{4}}\right) \partial / \partial b_{r}^{(1)}-\left(\zeta_{r_{2} r_{4}} / \zeta_{r_{3} r_{4}}\right) \partial / \partial b_{r}^{(2)}\right) & \text { if } i=r_{3}, \\ \epsilon_{r}\left(\zeta_{r_{1} r_{3}} / \zeta_{r_{3} r_{4}} \partial / \partial b_{r}^{(1)}+\zeta_{r_{2} r_{3}} / \zeta_{r_{3} r_{4}} \partial / \partial b_{r}^{(2)}\right) & \text { if } i=r_{4}\end{cases}
$$

$r_{1}, r_{2}, r_{3}, r_{4}$ are the vertices of $r$ in the increasing order. The coefficients at $\partial / \partial b_{r}^{(1)}$ and $\partial / \partial b_{r}^{(2)}$ are matrix elements of matrix $f_{4}$, in accordance with (13) and (9).

\footnotetext{
${ }^{7}$ The orientations of tetrahedra are actually important for us, but we will take them into account in another way, see Definition 3.
} 
Convention 2. Let $h$ be a homogeneous element of Grassmann algebra of degree $m$, and $d$ a homogeneous Grassmann differential operator of degree $n$. Then $d^{-1} h$ means any homogeneous element $f$ of Grassmann algebra (of degree $(m+n)$, of course) such that $d f=h$.

Theorem 3. The following function of Grassmann variables $a_{s}$ living on boundary 2-faces:

$$
\mathbf{T}=\int \cdots \int \prod_{\substack{\text { all } \\ \text { tetrahedra } r}} W_{r} \cdot\left(\prod_{\substack{\text { inner } \\ \text { vertices } i}} d_{i}^{\mathbf{a}}\right)^{-1} 1 \cdot\left(\prod_{\substack{\text { inner } \\ \text { vertices } i}} d_{i}^{\mathbf{b}}\right)^{-1} 1 \cdot \mathrm{d} \mathbf{b} \mathrm{d} \mathbf{a}_{\text {inner }}
$$

is the generating function for torsions $\tau_{\mathcal{C}}$, see $(14)$, in the sense that

$$
\mathbf{T}=\sum_{\mathcal{C}} \tau_{\mathcal{C}} \prod_{s \in \mathcal{C}} a_{s}
$$

In (18), d $\mathbf{a}_{\text {inner }}$ and d b stay for the products

$$
\mathrm{d} \mathbf{a}_{\text {inner }}=\prod_{\substack{\text { inner } \\ 2 \text {-faces } s}} \mathrm{~d} a_{s}, \quad \mathrm{~d} \mathbf{b}=\prod_{\substack{\text { all } \\ \text { tetrahedra } r}} \mathrm{~d} b_{r}^{(1)} \mathrm{d} b_{r}^{(2)},
$$

and the expressions (differential operator) $)^{-1} 1$ are defined according to Convention 2.

Proof. It is always possible to choose both $\left(\prod_{\substack{\text { inner } \\ \text { vertices } i}} d_{i}^{\mathbf{a}}\right)^{-1} 1$ and $\left(\prod_{\substack{\text { inner } \\ \text { vertices } i}} d_{i}^{\mathbf{b}}\right)^{-1} 1$ as Grassmann monomials - products of some Grassmann generators and a numeric factor. Then it can be seen that the numeric factor is exactly $\left(\operatorname{minor} f_{2}\right)^{-1}$ or $\left(\operatorname{minor} f_{4}\right)^{-1}$ respectively (compare formula (14)), where the rows in minor $f_{2}$ or the columns in minor $f_{4}$ correspond to the mentioned Grassmann generators. Then it is not hard to deduce that the factor at $\prod_{s \in \mathcal{C}} a_{s}$ in $\mathbf{T}$ is nothing but $\tau_{\mathcal{C}}$, and in passing we see that it does not depend on the choice of monomials. Nor will it change, of course, if we take a linear combination of monomials satisfying the same Grassmann differential equation

(differential operator) $f=1$.

Definition 4. We call the following function of Grassmann variables $a_{s}$ corresponding to boundary 2-faces $s$ generating function of invariants $I_{\mathcal{C}}^{(0)}$ :

$$
\mathbf{F}=\sum_{\mathcal{C}} I_{\mathcal{C}}^{(0)} \prod_{s \in \mathcal{C}} a_{s}
$$

It follows from Theorem 3 and formula (15) that

$$
\mathbf{F}=\frac{\prod_{\substack{\text { inner } \\ 2 \text {-faces } s}} \zeta_{s_{2} s_{3}}}{\prod_{\substack{\text { inner } \\ \text { edges } \ell}} \zeta_{\ell_{1} \ell_{2}} \prod_{\substack{\text { all } \\ \text { tetrahedra } r}} \zeta_{r_{3} r_{4}}} \cdot \mathbf{T} .
$$




\subsubsection{Opening the way to generalizations: Grassmann algebra relations corresponding to Pachner moves}

Writing the multicomponent invariants $\mathbf{F}$ (20) for the l.h.s. and r.h.s. of the Pachner move $2 \rightarrow 3$, see Fig. 1, we come to the following relation in Grassmann algebra:

$$
\frac{\zeta_{23}}{\zeta_{34} \zeta_{35}} \int \mathcal{W}_{1234} \mathcal{W}_{1235} \mathrm{~d} a_{123}=-\frac{1}{\zeta_{45}} \iiint \mathcal{W}_{1245} \mathcal{W}_{1345} \mathcal{W}_{2345} \mathrm{~d} a_{145} \mathrm{~d} a_{245} \mathrm{~d} a_{345}
$$

where we define

$$
\mathcal{W}_{r}=\iint W_{r} \mathrm{~d} b_{r}^{(1)} \mathrm{d} b_{r}^{(2)}
$$

and where we have also, of course, checked the sign separately. We also write $\mathcal{W}_{1234}, a_{123}$ and so on instead of more pedantic $\mathcal{W}_{(1234)}$ and $a_{(123)}$.

The Grassmann function $\mathcal{W}_{r}$ can be treated as the invariant $\mathbf{F}$ for just one tetrahedron $r$.

It is quite clear that Grassmann algebra relations like (21) can be taken themselves as a starting point for building manifold invariants and a topological quantum field theory, and there is, in principle, no need for the components of $\mathcal{W}_{r}$ to be related to torsions of any chain complex. This is the idea that we are going to explore.

\subsection{The deformed relations}

Our search for new Grassmann algebra relations associated with Pachner moves started with "deformations" of the weight $\mathcal{W}_{r}$ : what terms (if any) can be added to $\mathcal{W}_{r}$ so that relation (21) stays valid? Of course we have not yet found all possible deformations; the miracle is, however, that such deformations do exist, as shown by the results of our search, presented below.

\subsubsection{Deformation of degree 0}

We introduce the following deformation of the weight $\mathcal{W}_{r}$ for a tetrahedron $r=\left(r_{1} r_{2} r_{3} r_{4}\right)$ belonging to an oriented triangulated PL manifold $M$ with boundary:

$$
\tilde{\mathcal{W}}_{r} \stackrel{\text { def }}{=} \mathcal{W}_{r}+\epsilon_{r} \zeta_{r_{3} r_{4}} \alpha_{r}
$$

where $\epsilon_{r}$ is the tetrahedron orientation (see formula (12) and explanation after it), and $\alpha_{r}$ is some even element in the Grassmann algebra, depending on the tetrahedron $r$. Thus, $\alpha_{r}$ commutes with any other element, for instance, $\alpha_{r}$ may be a scalar $\in \mathbb{F}$, and this possibility looks (at this moment) the most natural. So, assuming $\alpha_{r} \in \mathbb{F}$, the term $\epsilon_{r} \zeta_{r_{3} r_{4}} \alpha_{r}$ in (23) has the degree 0, and we call $\tilde{\mathcal{W}}_{r}$ deformation of degree 0 of $\mathcal{W}_{r}$.

Remark 5. Both factors $\epsilon_{r}$ and $\zeta_{r_{3} r_{4}}$ are introduced in formula (23) because such definition of $\alpha_{r}$ clarifies formulas below, like (24)-(27).

The direct substitution of $\tilde{\mathcal{W}}_{r}$, given by the ansatz (23), in place of $\mathcal{W}_{r}$ in (21), using GAP [7] and our package PL [8] and assisted also by maxima [15], gives the following result: (21) holds for $\tilde{\mathcal{W}}_{r}$, provided the $\alpha$ 's in the r.h.s. of Pachner moves are expressed through the $\alpha$ 's in the 1.h.s. as follows:

$$
\begin{aligned}
& \zeta_{35} \alpha_{1235}-\zeta_{34} \alpha_{1234}=\zeta_{45} \alpha_{1245} \\
& \zeta_{25} \alpha_{1235}-\zeta_{24} \alpha_{1234}=\zeta_{45} \alpha_{1345} \\
& \zeta_{15} \alpha_{1235}-\zeta_{14} \alpha_{1234}=\zeta_{45} \alpha_{2345}
\end{aligned}
$$


Note that we have written the $\alpha$ 's corresponding to the r.h.s. of Pachner move also in the r.h.s. of (24), and we of course assume the same orientations for both sides of the move.

Equations (24) can be written in the following elegant form. First, it will be convenient for us to write $\alpha_{r}$ also as $\alpha_{\{i j k l\}}$, where $i, j, k$ and $l$ are the vertices of $r$ taken in an arbitrary order (recall that, according to Convention 1, we usually assume $i<j<k<l$ when we write $r=(i j k l)$ ). Now, choose an oriented edge $(k l)$; given also a consistent orientation of all tetrahedra, the link of $(\mathrm{kl})$ can be considered as made of oriented edges $(\mathrm{ij})$.

In an oriented edge $(i j)$, the order of vertices $i$ and $j$ determines its orientation, so this is an exception where Convention 1 does not work!

Definition 5. We say that a consistent system of $\alpha$ 's is given for a Pachner move $2 \leftrightarrow 3$ or $1 \leftrightarrow 4$, if

$$
\sum_{\begin{array}{c}
(i j) \text { in the } \\
\text { oriented link of }(k l) \\
\text { in the l.h.s. }
\end{array}} \zeta_{i j} \alpha_{\{i j k l\}}=\sum_{\begin{array}{c}
(i j) \text { in the } \\
\text { oriented link of }(k l) \\
\text { in the r.h.s. }
\end{array}} \zeta_{i j} \alpha_{\{i j k l\}} .
$$

If $(k l)$ is present in only one of the sides of Pachner move, then of course the sum in the corresponding side of (25) is zero. We also say that a consistent system of $\alpha$ 's is given for any triangulated oriented manifold $M$ with boundary, if

$$
\sum_{\substack{(i j) \text { in the } \\ \text { inted link of }(k l)}} \zeta_{i j} \alpha_{\{i j k l\}}=0
$$

for any inner (non-boundary) edge $(k l) \subset M$.

The three equations (24) have already the form (25) for edges 12, 13 and 23 respectively; other relations follow from these, like

$$
0=\zeta_{12} \alpha_{1245}-\zeta_{13} \alpha_{1345}+\zeta_{23} \alpha_{2345}
$$

for edge 45 .

Definition 6. Let a consistent system of $\alpha$ 's be given for a Pachner move $2 \leftrightarrow 3$ or $1 \leftrightarrow 4$, then for a tetrahedron $r=\left(r_{1} r_{2} r_{3} r_{4}\right)$, taking part in the move, we introduce its deformed Grassmann weight

$$
\tilde{W}_{r}=\exp \left(\Phi_{r}+\epsilon_{r} \zeta_{r_{3} r_{4}} \alpha_{r} b_{r}^{(2)} b_{r}^{(1)}\right) .
$$

Here $\Phi_{r}$ is the same as before, see formula (17).

Obviously, the analogue of (22) holds:

$$
\tilde{\mathcal{W}}_{r}=\iint \tilde{W}_{r} \mathrm{~d} b_{r}^{(1)} \mathrm{d} b_{r}^{(2)}
$$

Remark 6. The weight $\tilde{W}_{r}$ introduced in formula (28) coincides, essentially, with the second solution of pentagon equation in [10], with the constant multiplier $\mu$ omitted and in a different gauge. See [3, Subsection 5.3], where the transition from one gauge to the other is described for the case of "undeformed" weights - and this transition stays the same in the "deformed" case. 
Theorem 4. The weights defined according to (28) and (29) satisfy the following $2 \rightarrow 3$ relation:

$$
\frac{\zeta_{23}}{\zeta_{34} \zeta_{35}} \int \tilde{\mathcal{W}}_{1234} \tilde{\mathcal{W}}_{1235} \mathrm{~d} a_{123}=-\frac{1}{\zeta_{45}} \iiint \tilde{\mathcal{W}}_{1245} \tilde{\mathcal{W}}_{1345} \tilde{\mathcal{W}}_{2345} \mathrm{~d} a_{145} \mathrm{~d} a_{245} \mathrm{~d} a_{345}
$$

if the $\alpha$ 's form a consistent system for this move.

Moreover, the weights defined according to the same formulas, together with the operators $d_{i}^{\text {a }}$ and $d_{i}^{\mathbf{b}}$ given in Definitions 2 and 3 , satisfy the following $1 \rightarrow 4$ relation:

$$
\begin{aligned}
\frac{1}{\zeta_{34}} \tilde{\mathcal{W}}_{1234}= & -\frac{1}{\zeta_{15} \zeta_{45}} \int \cdots \int \tilde{W}_{1235} \tilde{W}_{1245} \tilde{W}_{1345} \tilde{W}_{2345} \cdot\left(d_{5}^{\mathbf{a}}\right)^{-1} 1 \cdot\left(d_{5}^{\mathbf{b}}\right)^{-1} 1 \\
& \times \mathrm{d} b_{1235}^{(1)} \mathrm{d} b_{1235}^{(2)} \mathrm{d} b_{1245}^{(1)} \mathrm{d} b_{1245}^{(2)} \mathrm{d} b_{1345}^{(1)} \mathrm{d} b_{1345}^{(2)} \mathrm{d} b_{2345}^{(1)} \mathrm{d} b_{2345}^{(2)} \\
& \times \mathrm{d} a_{125} \mathrm{~d} a_{135} \mathrm{~d} a_{145} \mathrm{~d} a_{235} \mathrm{~d} a_{245} \mathrm{~d} a_{345}
\end{aligned}
$$

if the $\alpha$ 's form a consistent system for this move.

Proof. We have already explained the formula (30). Similarly, formula (31) has been checked on a computer using our package PL [8].

Definition 7. We denote $\left(d_{5}^{\mathbf{a}}\right)^{-1} 1=u_{5}$ and $\left(d_{5}^{\mathbf{b}}\right)^{-1} 1=w_{5}$ and call them vertex weights for vertex 5 ; similarly below $u_{i}$ and $w_{i}$ for any vertex $i$. Each of them can be chosen as a monomial of degree one: $u_{i}$ containing Grassmann generator $a_{s}$ for some 2-face $s \ni i$, and $w_{i}$ containing $b_{r}^{(1 \text { or 2) }}$ for some tetrahedron $r \ni i$. If, additionally, $r \supset s$, we refer to both $u_{i}$ and $w_{i}$ as corresponding to tetrahedron $r$.

\subsubsection{Deformation of degree 4}

It turns out that there exists also another deformation $\tilde{\mathcal{W}}_{r}$ of our weight $\mathcal{W}_{r}$, where, instead of the term of degree 0 , a term of degree 4 is added to $\mathcal{W}_{r}$ :

$$
\tilde{\mathcal{W}}_{r} \stackrel{\text { def }}{=} \mathcal{W}_{r}+\epsilon_{r} \zeta_{r_{3} r_{4}} c_{r_{1} r_{2} r_{3} r_{4}} a_{r_{1} r_{2} r_{3}} a_{r_{1} r_{2} r_{4}} a_{r_{1} r_{3} r_{4}} a_{r_{2} r_{3} r_{4}}
$$

where

$$
c_{r_{1} r_{2} r_{3} r_{4}}=\prod_{1 \leq i<j \leq 4} \zeta_{r_{i} r_{j}}
$$

and $\epsilon_{r}= \pm 1$ is the same as in Subsection 2.2.1. For instance, in the following Theorem 5, $\epsilon_{(1234)}=\epsilon_{(1345)}=1$ and $\epsilon_{(1235)}=\epsilon_{(1245)}=\epsilon_{(2345)}=-1$.

The detailed study of the weight (32) is still in progress; here we just report about the result which we formulate as the following theorem.

Theorem 5. Tetrahedron Grassmann weights $\tilde{\mathcal{W}}_{r}$ defined according to (32) satisfy the same $2 \leftrightarrow 3$ relation (30).

Proof. Direct calculation.

Remark 7. The weight (32) appeared first in formula (11) of preprint [10], but in a different gauge, see again Remark 6 . 


\section{Four dimensions: deformed relations $3 \rightarrow 3$ and $2 \leftrightarrow 4$}

\subsection{Recalling the undeformed relations}

\subsubsection{The chain complex}

In four dimensions, the starting point for introducing the "undeformed" 4-simplex weight is again an exotic chain complex - a four-dimensional analogue of complex (5). We write out here its simplified - short - version, suited for studying Pachner moves $3 \rightarrow 3$ and $2 \leftrightarrow 4$. This will be enough for explaining then the current results of our symbolic calculations; a longer version of the complex will be presented in a separate paper [11].

We consider a triangulated orientable four-manifold $M$ with boundary and with the additional requirement that the triangulation has no inner vertices. Our short chain complex for such a manifold ${ }^{8}$ is:

$$
0 \longrightarrow \mathbb{C}^{N_{2}^{\prime}} \stackrel{f_{3}}{\longrightarrow} \mathbb{C}^{2 N_{3}} \stackrel{f_{4}}{\longrightarrow} \mathbb{C}^{3 N_{4}} \longrightarrow 0 .
$$

Here $N_{2}^{\prime}$ is the number of inner 2-faces, while $N_{3}$ and $N_{4}$ are the numbers of all 3 -faces and 4-simplices in $M$, respectively.

As before, all triangulation vertices have numbers $i$ from 1 to $N_{0}$ and complex "coordinates" $\zeta_{i}$ with the condition (6), and we again use notation (7) for their differences.

Remark 8. Again, like in the three-dimensional case, the numbering of mappings in (33) begins from $f_{3}$ because notations $f_{1}$ and $f_{2}$ (and actually $f_{5}$ and $f_{6}$ ) are reserved for longer complexes.

The spaces in (33) are much like those in (5). Again, we begin with auxiliary spaces: $W_{2}$ whose basis is formed of all pairs $(s, i)$, where $s$ is now an inner 2-face and $i \in s$ - its vertex, $W_{3}$ whose basis is formed of all pairs $(r, i)$, where $r$ is a 3 -face and $i \in r$-its vertex, and $W_{4}$ whose basis is formed of all pairs $(u, i)$, where $u$ is a 4 -simplex and $i \in u$-its vertex. Thus, $\operatorname{dim} W_{2}=3 N_{2}^{\prime}, \operatorname{dim} W_{3}=4 N_{3}$, and $\operatorname{dim} W_{4}=5 N_{4}$. We use notations $x_{s, i}, y_{r, i}$ or $z_{u, i}$ for coordinates of a vector $x \in W_{2}, y \in W_{3}$ or $z \in W_{4}$ respectively.

Then we introduce spaces $V_{2} \subset W_{2}$ and $V_{3} \subset W_{3}$ consisting of vectors whose coordinates obey the same relations (8) and (9) as in the three-dimensional case, and the space $V_{4} \subset W_{4}$ consisting of vectors $z$ whose coordinates also obey similar relations:

$$
\begin{aligned}
& z_{u, i}+z_{u, j}+z_{u, k}+z_{u, \ell}+z_{u, m}=0, \\
& \zeta_{i} z_{u, i}+\zeta_{j} z_{u, j}+\zeta_{k} z_{u, k}+\zeta_{\ell} z_{u, \ell}+\zeta_{m} z_{u, m}=0
\end{aligned}
$$

for every 4-simplex $u$ with vertices $i, j, k, \ell$ and $m$.

In the same way as in the three-dimensional case, we take for a vector $x \in V_{2}$ one coordinate in each (inner) 2-face $s$, namely coordinate corresponding to the vertex with the smallest number, to identify $V_{2}$ with $\mathbb{C}^{N_{2}^{\prime}}$, and similarly identify $V_{3}$ with $\mathbb{C}^{2 N_{3}}$ and $V_{4}$ with $\mathbb{C}^{3 N_{4}}$, taking two coordinates in each 3 -face or three coordinates in each 4 -simplex, respectively.

Linear mapping $f_{3}$ is defined by the old formulas (11), and linear mapping $f_{4}$ is also defined in a similar way. Namely, it makes the following $z_{u, i}$ from given $y_{r, i}$ :

$$
f_{4}:\left\{\begin{array}{l}
z_{u, i}=y_{(i j k \ell), i}-y_{(i j k m), i}+y_{(i j \ell m), i}-y_{(i k \ell m), i}, \\
z_{u, j}=y_{(i j k \ell), j}-y_{(i j k m), j}+y_{(i j \ell m), j}+y_{(j k \ell m), j}, \\
z_{u, k}=y_{(i j k \ell), k}-y_{(i j k m), k}-y_{(i k \ell m), k}+y_{(j k \ell m), k},
\end{array}\right.
$$

where 4-simplex $u$ has vertices $i<j<k<\ell<m$, and by $(i j k \ell)$ and so on we denote the 3 -faces of $u$ containing the indicated vertices.

\footnotetext{
${ }^{8}$ Of course, complex (33) can be written out also for a triangulation having inner vertices, but in such case (33) is not enough to obtain nonzero torsions.
} 
Theorem 6. The chain (33) of vector spaces and linear mappings defined as above is indeed a chain complex, i.e.,

$$
f_{4} \circ f_{3}=0 \text {. }
$$

Proof. Direct calculation.

\subsubsection{Changing the gauge}

Before constructing a Grassmann weight out of matrix $f_{4}$, we see it convenient to make a "gauge transformation" for this matrix. Namely, we multiply each column corresponding to each 3face $i j k \ell$ by $\zeta_{k \ell}$ (matrix $f_{4}$ has, of course, two columns for each 3 -face). We denote the resulting matrix $\tilde{f}_{4}$.

Accordingly, we divide each row of matrix $f_{3}$ by $\zeta_{k \ell}$, if it corresponds to a 3 -face $i j k \ell$. Additionally, we multiply each column of $f_{3}$ by $\zeta_{j k}$ if it corresponds to an inner 2-face $i j k$. The obtained matrix is denoted $\tilde{f}_{3}$. Obviously, the chain complex condition $\tilde{f}_{4} \tilde{f}_{3}=0$ still holds.

Below, Definitions 8 and 9 show how to construct Grassmann weights in order to interpret the torsions of complex (33) (in the new gauge) in terms of Grassmann algebra. Namely, in formula (34) for a 4-simplex weight, the three expressions in parentheses have matrix elements of the three rows of $\tilde{f}_{4}$ as coefficients at Grassmann variables (while multiplier $1 / \zeta_{45}$ is just for elegance, see Remark 9), and in formulas (35), the coefficients at partial derivatives are matrix elements of $\tilde{f}_{3}$.

\subsubsection{The formulas for weights}

We present now the undeformed 4-simplex weight, and the differential operators corresponding to 2-faces, in the gauge described above ${ }^{9}$. Instead of writing out the Grassmann weight $\mathcal{W}_{i j k l m}$ for a general 4 -simplex $(i j k l m)$, we write out just $\mathcal{W}_{12345}$ for readability; $\mathcal{W}_{i j k l m}$ is obtained by the obvious substitution $1 \mapsto i, \ldots, 5 \mapsto m$.

Definition 8. The undeformed Grassmann weight corresponding to 4-simplex (12345) is the following function of Grassmann variables $a_{i_{1} i_{2} i_{3} i_{4}}$ and $b_{i_{1} i_{2} i_{3} i_{4}}$ attached to each tetrahedron $\left(i_{1} i_{2} i_{3} i_{4}\right)-$ a 3 -face of (12345):

$$
\begin{aligned}
\mathcal{W}_{12345} \stackrel{\text { def }}{=} & \frac{1}{\zeta_{45}}\left(\zeta_{34} a_{1234}-\zeta_{35} a_{1235}+\zeta_{45} a_{1245}-\zeta_{45} a_{1345}\right) \\
& \times\left(\zeta_{34} b_{1234}-\zeta_{35} b_{1235}+\zeta_{45} b_{1245}+\zeta_{45} a_{2345}\right) \\
& \times\left(-\zeta_{14} a_{1234}-\zeta_{24} b_{1234}+\zeta_{15} a_{1235}+\zeta_{25} b_{1235}-\zeta_{45} b_{1345}+\zeta_{45} b_{2345}\right)
\end{aligned}
$$

Remark 9. The factor $1 / \zeta_{45}$ in (34) cancels out in all monomials obtained after expanding (34). So the weight (34) is bilinear in $\zeta$ 's and, moreover, the coefficient at each product of $a$ 's and/or $b$ 's has the form $\zeta_{i j} \zeta_{k l}$, where the subscripts may coincide. The total number of such terms (monomials with nonzero coefficients $\zeta_{i j} \zeta_{k l}$ ) in the expansion of (34) is 72, see [9, Appendix].

Definition 9. For a 2-face $s=(i j k)$, we introduce the differential operator $d_{i j k}$ as

$$
d_{i j k}=\sum_{\text {tetrahedra }} d_{t, s}
$$

where $d_{t, s}$ are the following operators, which we again prefer to write out putting numbers rather than letters in subscripts: we take tetrahedron $t=(1234)$ and its four faces, having in

\footnotetext{
${ }^{9}$ They have been already written in such form in preprint [9]; we only change the letter $W$ to $\mathcal{W}$, because our weight (34) is an analogue of (22) rather than (16). The situation with gauges in four dimensions is largely the same as in three dimensions, compare Remark 6.
} 
mind that, for an arbitrary tetrahedron $(i j k l)$ (remember that $i<j<k<l$ ), the substitution $1 \mapsto i, \ldots, 4 \mapsto l$ must be done. So, the operators are:

$$
d_{(1234), s}= \begin{cases}\left(\zeta_{23} / \zeta_{34}\right) \partial / \partial a_{1234}-\left(\zeta_{13} / \zeta_{34}\right) \partial / \partial b_{1234} & \text { if } s=(123), \\ -\left(\zeta_{24} / \zeta_{34}\right) \partial / \partial a_{1234}+\left(\zeta_{14} / \zeta_{34}\right) \partial / \partial b_{1234} & \text { if } s=(124) \\ \partial / \partial a_{1234} & \text { if } s=(134) \\ -\partial / \partial b_{1234} & \text { if } s=(234)\end{cases}
$$

\subsection{The deformed four-simplex weight and the relations $3 \rightarrow 3$ and $2 \leftrightarrow 4$}

Our deformed four-simplex Grassmann weight - an analogue of (23) - is introduced as follows. First, we need a "constant" (not depending on any simplices) odd element of Grassmann algebra, we denote it $e$. Then, we need an even element $\alpha_{\{i j k l m\}}$ for every four-simplex $(i j k l m)$, satisfying an analogue of relation (25), namely relation (37) below. The curly brackets around the subscripts of $\alpha$ mean the following: in order to write (37) in a simple way, it will be convenient for us to assume that the $\alpha$ 's depend on an unordered quintuple $\{i, j, k, l, m\}=\{j, i, k, l, m\}=$ $\cdots=\{m, l, k, j, i\}$, that is, an $\alpha$ does not change under a permutation of its indices.

Let there be a 2-face $(\mathrm{klm})$ in the l.h.s. or/and r.h.s. of a Pachner move. Then, given a consistent orientation (the same in both sides) of all 4-simplices, the link(s) of $(\mathrm{klm})$ can be considered as made of oriented edges $(i j)$. Recall that oriented edges form an exception from Convention 1 !

Definition 10. The deformed 4 -simplex weight, entering in a $3 \rightarrow 3$ or $2 \leftrightarrow 4$ Pachner move, is

$$
\tilde{\mathcal{W}}_{i j k l m}=\mathcal{W}_{i j k l m}+\alpha_{\{i j k l m\}} e,
$$

where the $\alpha$ 's are even elements of the Grassmann algebra satisfying the following relations for all 2 -faces $(k l m)$

$$
\sum_{\begin{array}{c}
(i j) \text { in the } \\
\text { oriented link of } k l m \\
\text { in the l.h.s. }
\end{array}} \zeta_{i j} \alpha_{\{i j k l m\}}=\sum_{\begin{array}{c}
(i j) \text { in the } \\
\text { oriented link of } k l m \\
\text { in the r.h.s. }
\end{array}} \zeta_{i j} \alpha_{\{i j k l m\}} \cdot
$$

If $(k l m)$ is present in only one of the sides of Pachner move, then of course the sum in the corresponding side of (37) iz zero.

Remark 10. Our formula (36) reveals some difference from its three-dimensional analogue (23). While the analogue of the factor $\zeta_{r_{3} r_{4}}$ is absent from (36) simply because of a different gauge, the role of $\epsilon_{r}$ is played in (36) by an object of apparently different nature - odd Grassmann element $e$. And the experimental fact that formulas (38) and (40) below hold for our weight $\tilde{\mathcal{W}}_{i j k l m}$ definitely deserves further research.

\subsubsection{Move $3 \rightarrow 3$}

Here is the description of the move $3 \rightarrow 3$ : the cluster of three 4-simplices (12345), (12346) and (12356), of which we think as being in the "l.h.s.", is replaced by the cluster of simplices (12456), (13456) and (23456) in the "r.h.s.". The boundary of either side consists of tetrahedra (1245), (1246), (1256), (1345), (1346), (1356), (2345), (2346), and (2356). The inner tetrahedra are, however, different: (1234), (1235) and (1236) in the l.h.s., and (1456), (2456) and (3456) in the r.h.s. Also, there is one inner 2-face (123) in the l.h.s., and one inner 2-face (456) in the r.h.s. 
Theorem 7. The following identity, corresponding naturally to the $3 \rightarrow 3$ Pachner move, holds:

$$
\begin{aligned}
& \int \tilde{\mathcal{W}}_{12345} \tilde{\mathcal{W}}_{12346} \tilde{\mathcal{W}}_{12356} w_{123} \frac{\mathrm{d} a_{1234} \mathrm{~d} b_{1234}}{\zeta_{34}} \frac{\mathrm{d} a_{1235} \mathrm{~d} b_{1235}}{\zeta_{35}} \frac{\mathrm{d} a_{1236} \mathrm{~d} b_{1236}}{\zeta_{36}} \\
& =\int \tilde{\mathcal{W}}_{12456} \tilde{\mathcal{W}}_{13456} \tilde{\mathcal{W}}_{23456} w_{456} \frac{\mathrm{d} a_{1456} \mathrm{~d} b_{1456}}{\zeta_{56}} \frac{\mathrm{d} a_{2456} \mathrm{~d} b_{2456}}{\zeta_{56}} \frac{\mathrm{d} a_{3456} \mathrm{~d} b_{3456}}{\zeta_{56}},
\end{aligned}
$$

where

$$
w_{123}=d_{123}^{-1} 1, \quad w_{456}=d_{456}^{-1} 1
$$

(for instance, $w_{123}=\zeta_{23}^{-1} \zeta_{34} a_{1234}$ and $w_{456}=-b_{1456}$, recall Convention 2$)$.

Proof. It can be checked that both sides of (38) cannot contain the $\alpha$ 's in the (total) degree more than one. The "constant" terms - not containing the $\alpha$ 's - form themselves the "undeformed" equation of preprint [9], which can be, and has been, verified separately on a computer. Then, it is not hard to see that, in order to prove the equality between the terms linear in $\alpha$ 's, it is enough to do so for just two following sets of $\alpha$ 's:

$$
\alpha_{\{12345\}}=\alpha_{\{12346\}}=\alpha_{\{12356\}}=\alpha_{\{12456\}}=\alpha_{\{13456\}}=\alpha_{\{23456\}}=1 \text {, }
$$

and

$$
\begin{aligned}
& \alpha_{\{12345\}}=\zeta_{6}, \quad \alpha_{\{12346\}}=\zeta_{5}, \quad \alpha_{\{12356\}}=\zeta_{4}, \\
& \alpha_{\{12456\}}=\zeta_{3}, \quad \alpha_{\{13456\}}=\zeta_{2}, \quad \alpha_{\{23456\}}=\zeta_{1} \text {. }
\end{aligned}
$$

This has also been done on a computer, using GAP [7] and our PL package [8].

\subsubsection{Move $2 \rightarrow 4$}

Next, we consider the following $2 \rightarrow 4$ move: the cluster of two 4 -simplices (12345) and (12346) is replaced by the cluster of four 4 -simplices (12356), (12456), (13456) and (23456). The boundary of both sides consists of tetrahedra (1235), (1236), (1245), (1246), (1345), (1346), (2345) and (2346).

In the l.h.s., there is one inner tetrahedron (1234) and no inner 2-faces.

In the r.h.s., there are six inner tetrahedra (1256), (1356), (1456), (2356), (2456) and (3456), and four inner 2 -faces (156), (256), (356) and (456). The $d$-operators for these 2-faces are, according to (35), as follows:

$$
\begin{array}{ll}
d_{156}=\frac{\partial}{\partial a_{1256}}+\frac{\partial}{\partial a_{1356}}+\frac{\partial}{\partial a_{1456}}, & d_{256}=-\frac{\partial}{\partial b_{1256}}+\frac{\partial}{\partial a_{2356}}+\frac{\partial}{\partial a_{2456}} \\
d_{356}=-\frac{\partial}{\partial b_{1356}}-\frac{\partial}{\partial b_{2356}}+\frac{\partial}{\partial a_{3456}}, & d_{456}=-\frac{\partial}{\partial b_{1456}}-\frac{\partial}{\partial b_{2456}}-\frac{\partial}{\partial b_{3456}} .
\end{array}
$$

So, one can check $^{10}$ that, for instance,

$$
a_{1256} b_{1256} a_{3456} b_{3456}
$$

is suitable as $w_{156,256,356,456}$ in formula (40) below.

\footnotetext{
${ }^{10}$ Recalling again Convention 2.
} 
Conjecture 8. The following identity, corresponding naturally to the $2 \rightarrow 4$ move, holds:

$$
\begin{aligned}
& \int \tilde{\mathcal{W}}_{12345} \tilde{\mathcal{W}}_{12346} \frac{\mathrm{d} a_{1234} \mathrm{~d} b_{1234}}{\zeta_{34}} \\
& \quad=-\zeta_{56} \int \tilde{\mathcal{W}}_{12356} \tilde{\mathcal{W}}_{12456} \tilde{\mathcal{W}}_{13456} \tilde{\mathcal{W}}_{23456} w_{156,256,356,456} \\
& \quad \times \frac{\mathrm{d} a_{1256} \mathrm{~d} b_{1256}}{\zeta_{56}} \frac{\mathrm{d} a_{1356} \mathrm{~d} b_{1356}}{\zeta_{56}} \frac{\mathrm{d} a_{1456} \mathrm{~d} b_{1456}}{\zeta_{56}} \frac{\mathrm{d} a_{2356} \mathrm{~d} b_{2356}}{\zeta_{56}} \frac{\mathrm{d} a_{2456} \mathrm{~d} b_{2456}}{\zeta_{56}} \frac{\mathrm{d} a_{3456} \mathrm{~d} b_{3456}}{\zeta_{56}} .
\end{aligned}
$$

Here, like in Theorem 7 , the weights $\tilde{\mathcal{W}}$ in $(40)$ are taken for the 4-simplices which are respectively in the l.h.s. and r.h.s.; the weight $w$ is taken for the inner 2-faces; and the integration is performed in $a$ and $b$ corresponding to the inner tetrahedra.

Partial verification. First, like in Theorem 7, both sides of (40) contain only terms of degree $\leq$ 1 in all $\alpha$ 's, and the "constant" parts form themselves the "undeformed" equation of [9] that has been verified directly and fully. Second, the equality between the parts linear in $\alpha$ 's have been checked not in full, but for some arbitrarily chosen values of $\zeta$ 's (assuming that we are working in the field $\mathbb{F}=\mathbb{Q}$ of rational numbers), such as

$$
\zeta_{1}=0, \quad \zeta_{2}=1, \quad \zeta_{3}=3, \quad \zeta_{4}=8, \quad \zeta_{5}=17, \quad \zeta_{6}=21 .
$$

Remark 11. The factor $\left(-\zeta_{56}\right)$ before the integral in the r.h.s. of $(40)$ is naturally interpreted as corresponding to the inner edge 56 in the cluster of four 4-simplices. There are of course no inner edges in any cluster of two or three 4-simplices considered in this paper.

Remark 12. Is both formulas (38) and (40), specific orderings/numberings of vertices $1, \ldots, 6$ were involved. For other orderings, similar formulas still hold, but we do not discuss it in this paper, where our modest aim is just to show the existence of such relations. Note that in a simpler three-dimensional situation, necessary arguments about (change of) vertex ordering are given in the proof of Theorem 10 .

\subsection{Conjectured invariant of moves $3 \rightarrow 3$ and $2 \leftrightarrow 4$}

Formulas (38) and (40) lead to a conjectured invariant of moves $3 \rightarrow 3$ and $2 \leftrightarrow 4$. We would like to formulate this as the following Conjecture 9, even if this conjecture looks somewhat preliminary at this stage of research, mainly because the question of choosing interesting consistent systems of $\alpha$ 's remains open. Note, however, that one possibility to satisfy equations (37) is to take all $\alpha$ 's simply equal to 1 !

Conjecture 9. The following expression, written for an arbitrary triangulated 4-manifold with boundary, remains invariant under moves $3 \rightarrow 3$ and $2 \leftrightarrow 4$ :

$$
\pm \prod_{\substack{\text { over inner } \\
\text { edges } i j}} \int \prod_{\substack{\text { over all } \\
4 \text {-simplices } i j k l m}} \tilde{\mathcal{W}}_{i j k l m} \cdot w \cdot \prod_{\begin{array}{c}
\text { over inner } \\
\text { tetrahedra } i j k l
\end{array}} \frac{\mathrm{da}_{\mathrm{ijkl}} \mathrm{db}_{\mathrm{ijkl}}}{\zeta_{k l}}
$$

where

$$
w=\left(\prod_{\substack{\text { over inner } \\ \text { 2-faces } i j k}} d_{i j k}\right)^{-1} 1 .
$$

The sign \pm in (41) corresponds to the fact that we did not specify the exact order in the products; most likely, there exists some elegant formula relating this order and this sign. 


\section{Again three dimensions: definition of a deformed invariant and nontriviality check}

\subsection{Some combinatorics before introducing the deformed invariant}

Definition 11. Let there be a finite set $X$ and its cover $\left\{S_{i}, i=1, \ldots, N\right\}$ consisting of its subsets $S_{i} \subset X$. Let there be also given an injection $f$ choosing an element in each of the covering sets:

$$
f: S_{i} \mapsto r_{i} \in S_{i}, \quad r_{i} \neq r_{j} \quad \text { if } \quad i \neq j .
$$

We call the mapping $f$ orderly if the following relation generates correctly a partial order for sets $S_{i}$ :

$$
S_{i} \leq S_{j} \quad \text { if } \quad f\left(S_{i}\right) \in S_{j} .
$$

Clearly, an orderly mapping $f$ sends $S_{k}$ into an element not belonging to any preceding set $S_{i}<S_{k}$. We denote

$$
R_{k}^{(f)}=S_{k} \backslash\left(\text { sets preceding } S_{k}\right) .
$$

Definition 12. An elementary move is the following change of an orderly mapping $f$ : change one image $f\left(S_{k}\right)$ to some other element $\tilde{r}_{k} \in R_{k}^{(f)}$; other images $f\left(S_{i}\right), i \neq k$, remain intact.

Remark 13. An elementary move may change the partial order on sets $S_{i}$, and thus, generally speaking, new subsets $R_{i}^{(h)} \subset S_{i}$ appear as its result, where $h$ denotes the result of applying the above elementary move to $f$. It does not, however, change $R_{k}^{(f)}=R_{k}^{(h)}$, because it does not affect the order of sets preceding $S_{k}$. So, the inverse to elementary move is also elementary move.

Lemma 1. For a given cover $\left\{S_{i}, i=1, \ldots, N\right\}$ of a finite set $X$, any orderly mapping $f$ can be transformed into any other orderly mapping $g$ by a sequence of elementary moves.

Proof. Induction in $N$ - the number of sets $S_{i}$. For $N=1$, the theorem obviously holds.

For arbitrary $N$, denote $S_{m}$ a maximal $S_{i}$, with respect to the partial order determined by mapping $f$. Then $R_{m}^{(f)} \subset S_{m}$ is its part not belonging to any other $S_{i}$. Consider the set $X^{\prime}=X \backslash R_{m}^{(f)}$, its cover of cardinality $N-1$ consisting of all $S_{i}$ except $S_{m}$, and restrictions of $f$ and $g$ on this cover. Due to induction hypothesis, the restriction of $f$ can be transformed into the restriction of $g$ by elementary moves. This means also that $f$ can be transformed into the mapping $\tilde{g}$ which is by definition the same as $g$ except for, maybe, just one image, $\tilde{g}\left(S_{m}\right) \stackrel{\text { def }}{=}$ $f\left(S_{m}\right)$.

Finally, changing $g$ to $\tilde{g}$ is also an elementary move, because $R_{m}^{(g)} \supset R_{m}^{(f)}$, so $g\left(S_{m}\right)$ is changed within $R_{m}^{(g)}$.

\subsection{The deformed multicomponent invariant of a three-manifold with triangulated boundary}

For an inner vertex $i$ in a triangulated orientable three-manifold $M$ with nonempty boundary $\partial M$, let $S_{i}$ be the set containing all tetrahedra in the star of $i$ as its elements, and $X$ be the union of all $S_{i}$. We are going to choose a tetrahedron in each $S_{i}$ using an orderly mapping $f$ in the sense of Definition 11.

We can construct $f$ as follows: let $r$ be any tetrahedron in the triangulation having at least one boundary (i.e., such that there is no tetrahedron on its other side) 2 -face. Then we remove $r$ from the triangulation. If, as a result of this, a vertex $i$ uncloses - ceases to be inner, we put $f\left(S_{i}\right)=r$. Then we repeat this step until there remain no inner vertices. 
Remark 14. If $S_{i}$ precedes $S_{j}$ in the sense of Definition 11, then vertex $i$ uncloses in this process after vertex $j$.

Remark 15. Let there also be a marked PL ball $B$ made of some tetrahedra in the triangulation, containing no inner vertices, in the sense that all its vertices are in $\partial B$. Then the above construction of $f$ can obviously be done without removing tetrahedra in $B$. We say then that $f$ avoids tetrahedra in $B$.

Definition 13. We say that we have chosen vertex weights according to orderly mapping $f$ if, for each inner vertex $i$, we have chosen $u_{i}$ and $w_{i}$ corresponding to the tetrahedron $f\left(S_{i}\right)$, see Definition 7.

Theorem 10. The following function of Grassmann variables and coordinates $\zeta_{i}$, considered up to an overall sign, is an invariant of a three-dimensional manifold $M$ with a fixed triangulation of its boundary $\partial M$ :

$$
\begin{aligned}
\mathbf{G}= & \frac{\prod_{\substack{\text { inner } \\
2 \text {-faces } s}} \zeta_{s_{2} s_{3}}}{\prod_{\substack{\text { inner } \\
\text { edges } \ell}} \zeta_{\ell_{1} \ell_{2}} \prod_{\substack{\text { all } \\
\text { tetrahedra } r}} \zeta_{r_{3} r_{4}}} \cdot \int \cdots \int \exp \left(\mathbf{b}^{\mathrm{T}} f_{3} \mathbf{a}+\mathbf{b}^{\mathrm{T}} C \mathbf{b}\right) \\
& \times \prod_{\substack{\text { inner } \\
\text { vertices } i}} u_{i} \cdot \prod_{\substack{\text { inner } \\
\text { vertices } i}} w_{i} \cdot \mathrm{d} \mathbf{b} \mathrm{d} \mathbf{a} .
\end{aligned}
$$

Here matrix $f_{3}$ is the same as in Subsection 2.1, and matrix $C$ is made of blocks

$$
\epsilon_{r}\left(\begin{array}{cc}
0 & \zeta_{r_{3} r_{4}} \\
-\zeta_{r_{3} r_{4}} & 0
\end{array}\right)
$$

where both rows and columns correspond to $b_{r}^{(1)}$ and $b_{r}^{(2)}$, in this order; vertex weights $u_{i}$ and $w_{i}$ are chosen according to any orderly mapping $f$.

Remark 16. Both Grassmann variables and coordinates $\zeta_{i}$ on which $\mathbf{G}$ depends belong, of course, to the boundary $\partial M$.

First, we prove the following lemma.

Lemma 2. Elementary moves, changing the orderly mapping $f$, do not affect the function $\mathbf{G}$ defined according to (43).

Proof. Consider an elementary move changing $f\left(S_{k}\right)$. Consider, in the situation before the move, the chain of all $S_{i}$ preceding $f\left(S_{k}\right)$, and let $S_{i_{1}}$ be the minimal element in this chain. If there are more than 4 tetrahedra ${ }^{11}$ in $S_{i_{1}}$, we, using a suitable sequence of Pachner moves $2 \rightarrow 3$ within the star of $i_{1}$ (each lessening the number of tetrahedra in the actual star of $i_{1}$ by one) and the final move $4 \rightarrow 1$, remove the vertex $i_{1}$ from triangulation; as there is exactly one vertex weight $u_{i_{1}}$ and one $w_{i_{1}}$ within the star, it can be seen that the moves can be chosen in such way that formula (30) can be applied to all moves $2 \rightarrow 3$ and formula (31) - to the final move. Then we do the same for the second element $S_{i_{1}}$ and so on, and finally we do this for $S_{k}$ itself.

The same can obviously be done for the situation after the move, with the very same final triangulation. So, there is a chain of transformations connecting the two choices of $f$ and not affecting $\mathbf{G}$.

\footnotetext{
${ }^{11}$ With our definition of triangulation, there might be also just 2 tetrahedra in the star; we leave this easy case to the reader.
} 


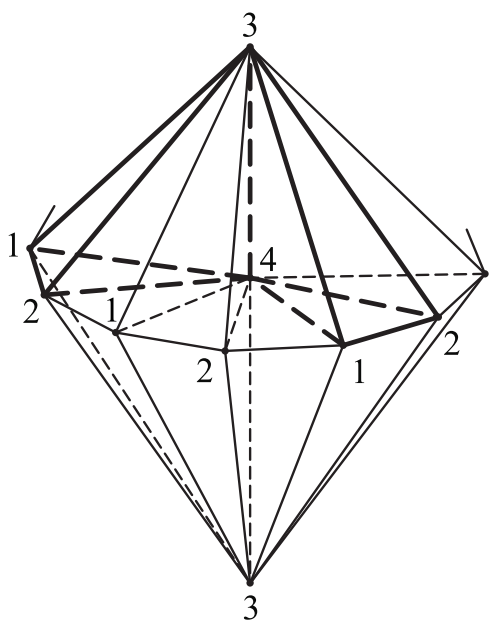

Figure 3. Triangulated lens space with a chain of two tetrahedra.

Proof of Theorem 10. Any triangulation of the interior of $M$ can be transformed into any other triangulation by a sequence of relative Pachner moves, i.e., moves leaving the boundary triangulation intact. As this has been explained in detail in [5, Section 2], here we only note that, although the boundary in [5] was just a specially triangulated torus, the techniques generalize directly to the case of a general boundary.

We want now to apply Theorem 4 to each of these Pachner moves. First, comparing (43) and (44) with (28), we see that this will work if all $\alpha$ 's in Theorem 4 are chosen to equal -2 (condition 25 is then obviously satisfied). Second, we must ensure the right number of multipliers $u_{i}$ living on 2 -faces and $w_{i}$ living in tetrahedra involved in the move, namely zero for moves $2 \leftrightarrow 3$ and $1 \rightarrow 4$, and one $u_{5}$ and one $w_{5}$ for $4 \rightarrow 1$, in accordance with (30) and (31). This is, however, easy to do having in mind Remark 15: for all moves except $4 \rightarrow 1$, construct $f$ avoiding all tetrahedra that are to be replaced, and for $4 \rightarrow 1$ - avoiding three of them. The possible change of $f$ is, of course, justified by Lemmas 1 and 2 .

And third, formulas (30) and (31) may seem to require a specific ordering of vertices involved in any Pachner move. This question is solved by re-formulating our results in the "symmetric" gauge of [10], in which the Grassmann weight of a tetrahedron cannot depend on the order of vertices in any way except its overall sign (recall again that the transition from one gauge to the other is described in [3, Subsection 5.3]).

Remark 17. We see thus that the following consistent system of $\alpha$ 's corresponds to invariant (43):

$$
\alpha_{r}=-2 \text { for all tetrahedra } r \text {. }
$$

It is of course interesting how to introduce an invariant with more general $\alpha$ 's than just all the same constant. We leave this question for further work.

\subsection{Example calculation: triangulated lenses without tubular neighborhoods of unknots}

Consider a three-dimensional lens space $L(p, q)$, represented in a standard form of a bipyramid whose upper half-surface is glued to its lower half-surface after a rotation through angle $2 \pi q / p$ around the vertical axis (see, for instance, textbook [6]). Let then the bipyramid be divided into $4 p$ tetrahedra, all with the same vertices 1, 2, 3, 4. A fragment of such triangulation is shown in Fig. 3. Then we choose an integer $n \neq 0 \bmod p$, and two tetrahedra in this triangulation 


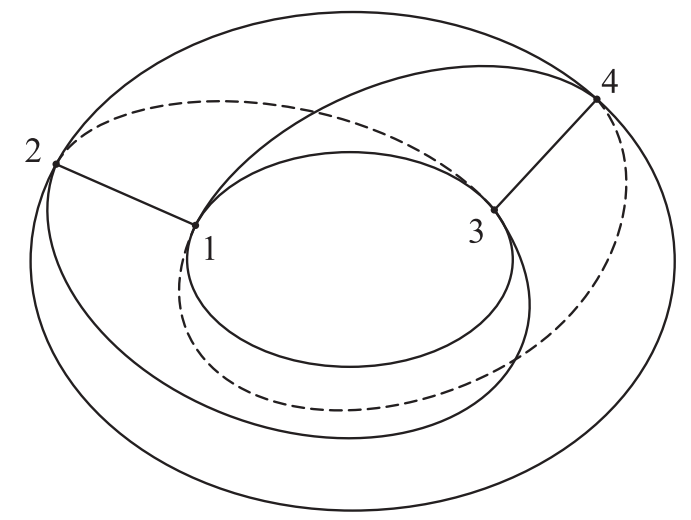

Figure 4. The chain of two tetrahedra.

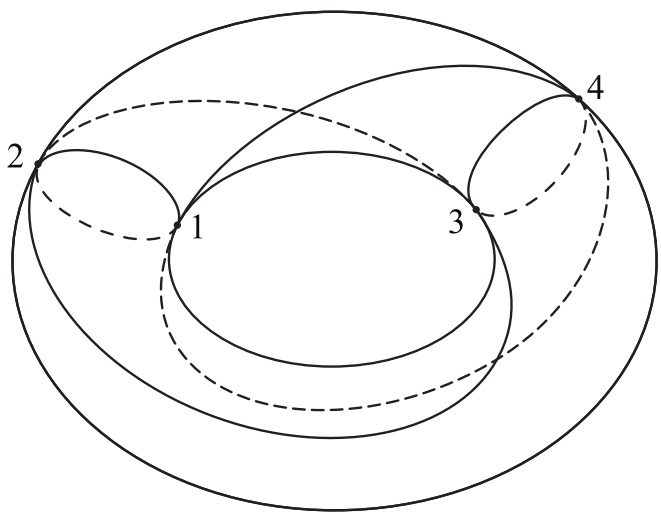

Figure 5. After doubling edges 12 and 34, the boundary of what remains of lens space becomes a torus triangulated in 8 triangles.

obtained one from another by a rotation through angle $2 \pi n / p$. In Fig. 3, these are shown in boldface lines, for the case $n=2$.

We thus obtain a chain of two tetrahedra in $L(p, q)$, of the form pictured in Fig. 4 . Removing the interiors of these tetrahedra from $L(p, q)$, and doubling common for two tetrahedra edges 12 and 34 as shown in Fig. 5, we obtain the lens space without a tubular neighborhood of an unknot representing a 1-cycle determined by the number $n$ above. Here an "unknot" in a closed 3manifold $M$ is characterized by the property that it can be represented by an unknotted line when $M$ is represented as a 3-ball with its surface glued in a proper way to itself. We denote the obtained 3-manifold with triangulated torus boundary as $\tilde{L}$.

Next, we can calculate the invariant function $\mathbf{G}(43)$ for $\tilde{L}$, denoted $\mathbf{G}_{\tilde{L}}$. As there are no inner vertices in our triangulation, (43) reduces to

$$
\mathbf{G}_{\tilde{L}}=\frac{\prod_{\substack{\text { inner } \\ 2 \text {-faces } s}} \zeta_{s_{2} s_{3}}}{\prod_{\substack{\text { inner } \\ \text { edges } \ell}} \zeta_{\ell_{1} \ell_{2}} \prod_{\substack{\text { all } \\ \text { tetrahedra } r}} \zeta_{r_{3} r_{4}}} \cdot \int \cdots \int \exp \left(\mathbf{b}^{\mathrm{T}} f_{3} \mathbf{a}+\mathbf{b}^{\mathrm{T}} C \mathbf{b}\right) \mathrm{d} \mathbf{b} \mathrm{d} \mathbf{a} .
$$

Our modest aim in this paper is just to show that our deformed invariants are nontrivial, that is, take some interesting values ${ }^{12}$ that, we hope, deserve further investigation. We think that, at this stage, it is enough to present the results for the monomial in $\mathbf{G}_{\tilde{L}}$ of degree zero in

\footnotetext{
${ }^{12}$ Even for all $\alpha=1$.
} 
anticommuting variables; we denote it $\mathrm{G}_{\tilde{L}}$. The essential point with $\mathrm{G}_{\tilde{L}}$ is that its calculation really involves the deformation, that is, it would vanish if we took all $\alpha$ 's equal to zero.

We simply present the following tables of directly calculated values of $\mathrm{G}_{\tilde{L}}$. We made use of the fact that the integral in (45) is the Pfaffian of the quadratic form in the exponent. Also, it was enough for us to do calculations for specific values of $\zeta$ 's using the already cited GAP system and our package PL, although it makes little doubt that general formulas for a Pfaffian with a regular structure can be derived.

$L(7,1)$ :

\begin{tabular}{c|c|c}
$n$ & $\zeta_{1}=1, \zeta_{2}=2, \zeta_{3}=3, \zeta_{4}=4$ & $\zeta_{1}=1, \zeta_{2}=2, \zeta_{3}=4, \zeta_{4}=3$ \\
\hline 1 & 153 & 92 \\
\hline 2 & 313 & 324 \\
\hline 3 & 381 & 452
\end{tabular}

\begin{tabular}{lc|c|c} 
& $n$ & $\zeta_{1}=1, \zeta_{2}=2, \zeta_{3}=3, \zeta_{4}=4$ & $\zeta_{1}=1, \zeta_{2}=2, \zeta_{3}=4, \zeta_{4}=3$ \\
\hline 1 & 12 & 61 \\
\hline $2(7,2):$ & 108 & 39 \\
\hline & 2 & 153 & 92
\end{tabular}

\begin{tabular}{lc|c|c} 
& $n$ & $\zeta_{1}=1, \zeta_{2}=2, \zeta_{3}=3, \zeta_{4}=4$ & $\zeta_{1}=1, \zeta_{2}=2, \zeta_{3}=4, \zeta_{4}=3$ \\
\hline \multirow{2}{*}{$L(7,3):$} & 39 & 108 \\
\hline & 2 & 92 & 153 \\
\hline & 3 & 61 & 12
\end{tabular}

Remark 18. Recall that every value of $G_{\tilde{L}}$ in these three tables is defined up to a sign.

Remark 19. $L(7,3)$ was here, of course, just for controle, as it is known to be homeomorphic to $L(7,2)$ and, moreover, a PL homeomophism can be described explicitly in a simple and direct way as disassembling a bipyramid representing one of these spaces into a set of tetrahedra and then assembling them back into another bipyramid, see, e.g., textbook [6].

\section{Discussion}

Our hope is that our Pacher-move-like algebraic relations will lead to constructing new topological quantum field theories (TQFT's). As they will be based on the Grassmann-Berezin calculus, they can be called "fermionic TQFT's". This is, of course, especially interesting in four dimensions.

It would be interesting to relate our research to ideas of the paper [1]. More specifically, it is interesting to know whether the formulas in [1] can be extended/deformed to incorporate a case outside of a pure Reidemeister torsion, as well as four-dimensional manifolds.

On the other side, we considered in the present paper only the case where a "coordinate" $\zeta_{i}$ is put in correspondence to a vertex $i$, and not a more general case where a universal cover of the manifold is involved and different coordinates correspond to different lifts of a vertex. In terms of [1], this corresponds to considering a trivial flat connection and a trivial bundle over the manifold. So, one direction of our further research may involve our "deformed" Pacher-movelike algebraic relations together with nontrivial flat connections. In the "undeformed" case and a slightly different specific theory, this was done in papers $[13,14]$.

One more very important generalization may be made through considering general consistent systems of $\alpha$ 's (see (25) and (37)). This is expected to be related to some interesting homological problems. Also, some parameter counting suggests that in the four-dimensional case, interesting deformations of degree 1 in variables $a$ and $b$ are expected to exist, which may be even more interesting than the deformation (36). 
Finally, we emphasize that our calculations in Subsection 4.3 simply show the nontriviality of invariants arising from our "deformed" relations. The nature of these invariants and possible relations to existing theories are still to be investigated, and again especially in four dimensions.

\section{Acknowledgements}

This paper has been written with partial financial support from Russian Foundation for Basic Research, Grant no. 10-01-00088-a, and a grant from the Academic Senate of Moscow State University of Instrument Engineering and Computer Sciences. I also thank the referees for their constructive and helpful comments.

\section{References}

[1] Barrett J.W., Naish-Guzman I., The Ponzano-Regge model, Classical Quantum Gravity 26 (2009), 155014, 48 pages, arXiv:0803.3319.

[2] Berezin F.A., Introduction to superanalysis, Mathematical Physics and Applied Mathematics, Vol. 9, D. Reidel Publishing Company, Dordrecht, 1987.

[3] Bel'kov S.I., Korepanov I.G., A matrix solution of the pentagon equation with anticommuting variables, Theoret. and Math. Phys. 163 (2010), 819-830, arXiv:0910.2082.

[4] Bel'kov S.I., Korepanov I.G., Martyushev E.V., A simple topological quantum field theory for manifolds with triangulated boundary, arXiv:0907.3787.

[5] Dubois J., Korepanov I.G., Martyushev E.V., A Euclidean geometric invariant of framed (un)knots in manifolds, SIGMA 6 (2010), 032, 29 pages, math.GT/0605164.

[6] Fomenko A.T., Matveev S.V., Algorithmic and computer methods for three-manifolds, Mathematics and its Applications, Vol. 425, Kluwer Academic Publishers, Dordrecht, 1997.

[7] GAP - Groups, Algorithms, Programming - a system for computational discrete algebra, http://www. gap-system.org/.

[8] Korepanov A.I., Korepanov I.G., Sadykov N.M., PL: Piecewise-linear topology using GAP, http://sf .net/ projects/plgap/.

[9] Korepanov I.G., Algebraic relations with anticommuting variables for four-dimensional Pachner moves $3 \rightarrow 3$ and $2 \leftrightarrow 4$, arXiv:0911.1395.

[10] Korepanov I.G., Two deformations of a fermionic solution to pentagon equation, arXiv:1104.3487.

[11] Korepanov I.G., Sadykov N.M., Four-dimensional Grassmann-algebraic TQFT's, work in progress.

[12] Lickorish W.B.R., Simplicial moves on complexes and manifolds, in Proceedings of the Kirbyfest (Berkeley, CA, 1998), Geom. Topol. Monogr., Vol. 2, Geom. Topol. Publ., Coventry, 1999, 299-320, math.GT/9911256.

[13] Martyushev E.V., Euclidean simplices and invariants of three-manifolds: a modification of the invariant for lens spaces, Izv. Chelyabinsk. Nauchn. Tsentra 2003 (2003), no. 2 (19), 1-5, math.AT/0212018.

[14] Martyushev E.V., Euclidean geometric invariants of links in 3-sphere, Izv. Chelyabinsk. Nauchn. Tsentra 2004 (2004), no. 4 (26), 1-5, math.GT/0409241.

[15] Maxima, a computer algebra system, http://maxima.sourceforge.net/.

[16] Pachner U., P.L. homeomorphic manifolds are equivalent by elementary shellings, European J. Combin. 12 (1991), 129-145.

[17] Turaev V.G., Introduction to combinatorial torsions, Lectures in Mathematics ETH Zürich, Birkhäuser Verlag, Basel, 2001. 